\title{
Thermal conductivity measurement of porous silicon by pulsed-photothermal method
}

\author{
E. Amin-Chalhouba , N. Semmar , L. Coudron ${ }^{b}$, G. Gautier ${ }^{b}$, C. Boulmer-Leborgne, \\ A. Petit ${ }^{a}$, M. Gaillard ${ }^{a}$, J. Mathias ${ }^{a}$, E. Millon ${ }^{a}$ \\ a GREMI, UMR 6606, Université d'Orléans-CNRS, 14 rue d'Issoudun, BP 6744, 45067 Orléans, France \\ b LMP, Université de Tours/ST-Microelectronics, 16 rue Pierre et Marie curie, 37071 Tours, France
}

Corresponding author: nadjib.semmar@univ-orleans.fr

\begin{abstract}
Thermal properties of two types of porous silicon are studied using the pulsed-photothermal method (PPT). This method is based on a pulsed-laser source in the nanosecond regime. A 1D analytical model is coupled with the PPT technique in order to determinate thermal properties of the studied samples (thermal conductivity and volumetric heat capacity).

At first, a bulk single crystal silicon sample and a titanium thin film deposited on a single crystal silicon substrate are studied in order to validate the PPT method. Porous silicon samples are elaborated with two different techniques, the sintering technique for macroporous silicon and electrochemical etching method for mesoporous silicon. Metallic thin films are deposited on these two substrates by magnetron sputtering. Finally, the thermal properties of macroporous (30\% of porosity and pores diameter between $100 \mathrm{~nm}$ and $1000 \mathrm{~nm}$ ) and mesoporous silicon (30\% and $15 \%$ of porosity and pores diameter between $5 \mathrm{~nm}$ and $10 \mathrm{~nm}$ ) are determined in this work and it is found that thermal conductivity of macroporous $\left(73 \mathrm{~W} \cdot \mathrm{m}^{-1} \cdot \mathrm{K}^{-1}\right)$ and mesoporous (between 80 and $50 \mathrm{~W} \cdot \mathrm{m}^{-1} \cdot \mathrm{K}^{-1}$ ) silicon is two times lower than the single crystal silicon $\left(140 \mathrm{~W} \cdot \mathrm{m}^{-1} \cdot \mathrm{K}^{-1}\right)$.
\end{abstract}

Keywords: Porous silicon, thermophysical properties, laser heating, pulsed photothermal method.

\section{Introduction}

Since the porous silicon (porous $\mathrm{Si}$ ) has very good properties of electroluminescence and photoluminescence in the visible and IR spectra [1, 2], it is now an important candidate for application in the optoelectronic and microelectronic fields [3] mainly due to its relatively wide specific surface. The wavelength of the emitted light varies as a function of the porosity of the sample [4]. Moreover, the porous Si refractive index can be modulated depending on the porosity. These properties are very interesting in photonics which applications can extend in many fields like gas detectors, anti-reflection coatings, waveguides, photonic crystals [5]... Other applications of porous $\mathrm{Si}$ are in the biology field, such as bio-detector since it is a bio-compatible material. Systems of bio-detection based on wavelength shift in a Fabry-Perot cavity are made with thin films of porous Si [5].

Furthermore, porous Si substrate is a good thermal insulator and its structures are more mechanically stable than single crystal silicon ( $\mathrm{sc}-\mathrm{Si}$ ) leading to more applications in micro-systems, for example thermopile fabrication $[6,7,8]$. Since it is widely used in various technological fields, it is then very important to determine the thermal conductivity especially at the micrometer scale. The thermal properties of porous $\mathrm{Si}$ are still not very well defined depending mainly on the fabrication process and the pore geometry.

Recent studies have demonstrated that the thermal conductivity of porous Si does not vary linearly as a function of the porosity [5] using an IR thermography based method. Simulations were made in order to establish the relationship between the size of pores and the thermal conductivity [9]. Using the photoacoustic method, it has been shown that the thermal conductivity of porous $\mathrm{Si}$ is twice smaller than the sc-Si one [10].Many methods can be employed in order to detremine the thermal properties of thin films or substrates. 
The thermoreflectance or thermoreflectance thermography tehcniques are used for example to detrmine the thermal conductivity of silicon films and isotopicaly pure silicon [11, 12]. The electrical resistance thermometry or the 3 omega methods can also be cited as reliable techniques for a multi-layer thermal properties investigations [13, 14]. In this work, the apparent thermal conductivity of two types of porous Si is determined by the photothermal nanosecond method $[15,16]$. The first one is fabricated with the sintering technique with pores diameter varying between $100 \mathrm{~nm}$ and $1000 \mathrm{~nm}$ [17] and the other one has its pores diameter very small, varying between $5 \mathrm{~nm}$ and $10 \mathrm{~nm}$ and it has been elaborated by electrochemical etching [18]. The advantage of this method is the ability to compare the thermal conductivity of porous Si having two different sizes of pores in order to estimate the relation between these parameters. The original contribution consists mainly in applying a non destructive method on complex surfaces, including a metallic thin coating, in direct connection with the concerned microelectronic applications.

\section{Experimental set-up for photothermal method}

The experimental set-up is based on the nanosecond pulsed photothermal method. This method consists on the detection of the IR radiations emitted from the surface of a sample after its interaction with a pulsed laser beam. The sample, being heated by a UV KrF laser pulse ( $\lambda=248 \mathrm{~nm}, \tau=27 \mathrm{~ns})$, emits IR thermal waves that are focalized by two parallel off-axis paraboloid mirrors into the active area $(0.25 \mathrm{~mm}$ diameter) of an IR detector. The IR detector is cooled with liquid nitrogen and has a wide spectral range from $2 \mu \mathrm{m}$ to $12 \mu \mathrm{m}(300 \mathrm{~K}$ to $3000 \mathrm{~K})$ with an integrated preamplifier of $100 \mathrm{MHz}$. The output signal of the IR detector is read by a numerical oscilloscope ( $500 \mathrm{MHz}$ bandwidth) that can give simultaneously the time distribution of the KrF laser beam (UV) and the IR signal variations at the nanosecond time scale.

The choice of the $\mathrm{KrF}$ laser as an excitation source is mainly due to the wavelength of the emitted radiations and also its interaction time. Most of metals have an important absorption coefficient in the UV range, the $\mathrm{KrF}$ laser beam is then absorbed and heats the surface of the sample. The small interaction time permits to probe the extreme surface of the sample (several hundreds of nanometers) which makes this method very suitable for the thermal characterization of thin films. Finally, the use of UV radiations to heat the sample permits to avoid the perturbation of the IR thermal signal emitted from the surface of the sample.

Figure 1 outlines the complete measurement system, the dashed path represents the UV laser beam detected by a phototube and the dotted one corresponds to the IR radiation trajectory.

\section{Elaboration technique of porous silicon}

A n-doped (100) Single crystal silicon (sc-Si) and two types of porous $\mathrm{Si}$, macroporous silicon (macro-Si) and mesoporous silicon (meso-Si) are studied in this work.

The macro-Si sample (Figure 2, a) has pores diameter in the micrometer scale (100 nm-1000 nm) and a porosity of $30 \%$. This sample is elaborated by the sintering technique that consists in making fine grinding of the sample before compressing under high pressure and annealing in order to obtain a compact sample.

The meso-Si substrate (Figure 2, b) is prepared by the electrochemical etching technique. The porous silicon layers are formed by anodization of N-doped $(0,012-0,015 \Omega \cdot \mathrm{cm}) 6$ inches n-type single crystal silicon (100) oriented wafers with a thickness of $240 \mu \mathrm{m}$. The electrolytic etching is performed in a double tank electrochemical cell developed by AMMT Corporation (Figure 3). The electrolyte is based on a HF $(50 \%): \mathrm{H}_{2} \mathrm{O}$ : acetic acid $(4.63: 1.45: 2.14)$ mixture. The electrochemical etching is carried out with a constant current density of $93 \mathrm{~mA} . \mathrm{cm}^{-2}$ during $10 \mathrm{~min}$ in the dark. The porosity of the samples is estimated using weight measurements [19]. P-type or n-type doped silicon is known to produce mesoporous materials in HF solutions corresponding to pore diameters varying from 2 to $50 \mathrm{~nm}$ [20]. Moreover, the morphology is influenced by the crystal orientation with a preferred growth in the $<100>$ direction leading to columnar structures [21]. In our case, the porosity is about 30\%, the layer thickness is $50 \mu \mathrm{m}$ and the average pores diameter ranges from $5 \mathrm{~nm}$ to $10 \mathrm{~nm}$ with the presence of secondary branches. This observation is in good accordance with a previous work [22].

Notice that during this study, sc-Si and meso-si samples are n-dopoed with the same concentration level (5-10.10 $\left.{ }^{18} \mathrm{~cm}^{-3}\right)$, the electrochemical technique does not change the dopant concentration. As for the macro$\mathrm{Si}$, it is made of polycrystal silicon with different size of cristals, after the compressing and annealing phases, the mophology and size of cristallites changes [14]. As reported in [23, 14, 5, 13, 6, 24], for polycrystalline silicon thin films, the thermal conductivity is varying from 1 to $35 \mathrm{~W} \cdot \mathrm{m}^{-1} \cdot \mathrm{K}^{-1}$. It is however difficult to compare these results to the present case (macro-Si with $300 \mu \mathrm{m}$ thick). 


\section{Coating technique deposition}

With the pulsed-photothermal method, thermal properties of materials (bulk or multilayer samples) can be determined if the laser beam energy is absorbed homogeneously on the surface of the sample. But in our case, the complex surface of porous silicon does not allow an homogeneous absorption of the photons arriving on the surface. When the laser beam heats the surface, the interaction area is not very well defined either in the laser/phonon or laser/silicon case. In addition, when the UV beam irradiates the porous silicon, a signal of photoluminescence in the IR band is emitted [25]. The photoluminescence phenomenon masks the thermal signal, and it becomes very difficult to differentiate between photoluminescence and thermal radiations $(2-12 \mu \mathrm{m})$. For this reason, the deposition of a thin metallic layer on the surface of each porous sample is necessary. In fact, this metallic layer absorbs the UV beam, creating a uniform heat source, and transmits the photon energy toward the substrate by phonon vibrations.

Absorbing layer were deposited on samples by physical vapor deposition technique (PVD). A $200 \mathrm{~nm}$ thick film of titanium is deposited on (100) oriented single crystal silicon ( $\mathrm{sc}-\mathrm{Si}$ ) by ion beam PVD in a commercial device (Precision Etching Coating System)(Figure 4). An $\mathrm{Ar}^{+}$ion beam is used to erode the target. The sputtered metallic particles condensate on the sample to create the coating.

Metallic thin films are deposited on porous substrate by magnetron sputtering on a research device. The metallic targets were sputtered by the ionic species $\left(\mathrm{Ar}^{+}\right)$coming from a plasma created by a DC voltage. Titanium thin film is deposited on the meso-Si substrate (Figure 5, a) and tungsten thin film is deposited on the macro-Si (Figure 5, b). The use of tungsten instead of titanium is mainly due to the bad adhesion of titanium thin film on the macro-Si (higher roughness and higher pore sizes). Table 1 summarizes the characteritics of the different samples studied in this work.

The experimental protocol of thermal conductivity identification is composed of four steps: first, the thermal conductivity of sc-Si (sample 1) is measured to validate the measurement in the case of semi-infinite surface (i.e. without coating). Second, the method is validated in the case of multilayer sample, $\mathrm{Ti} / \mathrm{sc}-\mathrm{Si}$ (sample 2) in order to obtain the same value of thermal conductivity of sc-Si. In the third and fourth steps, measurements are done on $\mathrm{W} /$ macro-Si (sample 3) and $\mathrm{Ti} /$ meso-Si (sample 4) in order to determinate the thermal conductivity of the two types of porous silicon. Notice that the optical absorptivity coefficient of W is similar to Ti in the UV range and investigation of $\mathrm{W}$ thin film was already done by our group using the photothermal method [16].

\section{Results and discussion}

\subsection{Calibration}

Thermal properties of thin films are determined from the relaxation of the temporal surface temperature after one laser pulse. In order to deduce the surface temperature from the electrical signal of the IR sensor, a calibration process is necessary for each sample. In fact, the emissivity of each surface depends on the nature of the material, its surface state and thickness. For this purpose, an electrical resistance is located behind the sample holder in order to be in contact with the back side of the sample. Once the sample is heated by this resistance, it emits IR thermal radiations from the front of the surface. These radiations are measured by the IR detector. At the same time, a K-type thermocouple is put in contact with the sample. So the detector output variations (electrical voltage) can be plotted versus temperature values. Calibration curves are given in Figure 6. As indicated, sample 1 and sample 2 exhibit a similar thermal radiation because the emissivity of $\mathrm{Ti}$ and $\mathrm{Si}$ are both close to 0.5 in the spectral range 2-12 $\mu \mathrm{m}$. Due to the porosity, thermal radiations emitted from sample 3 and 4 are slightly under the previous ones. For example, at $250^{\circ} \mathrm{C}$, the amplitude of the IR signal emitted from the surface of sc-Si and $\mathrm{Ti} / \mathrm{sc}-\mathrm{Si}$ (samples 1 and 2) is $65 \pm 5 \mathrm{mv}$ which is more important than $50 \pm 5 \mathrm{mV}$ value, that corresponds to the signal emitted from $\mathrm{W} /$ macro-Si (sample 3).

The calibration method is performed in this work with the samples placed into an hemispherical cavity which amplifies the apparent emissivity of the samples. The cavity eliminates ambient radiation reflections while acting almost as a black body (due to multiple reflections between the specular cavity surface and the measured sample) so that the effective emissivity is increased. It also allows measurements to be taken under vacuum, thus the absorption of particles of the air especially $\mathrm{CO}_{2}$ and $\mathrm{H}_{2} \mathrm{O}$ can be avoided. 


\subsection{Validation of the model}

In the case of thin films, the time evolution of the apparent thermal effusivity $(\mathrm{e}(\mathrm{t}))$, taking into account the absorptivity of the material at $248 \mathrm{~nm}$ and the energy-time distribution of the laser pulse (in this study rectangular shape), is given by the following equation [26]:

$$
e(t)=\frac{Q}{T(t) \sqrt{\pi t}} \text { withe }=\sqrt{\kappa \cdot \rho c_{p}}
$$

The variation of surface temperature $\mathrm{T}(\mathrm{t})$ is obtained experimentally by the pulsed-photothermal method. $\mathrm{Q}$ is the absorbed laser beam energy density; $\kappa\left(\mathrm{W} \cdot \mathrm{m}^{-1} \cdot \mathrm{K}^{-1}\right)$ is the thermal conductivity, $\rho\left(\mathrm{kg} \cdot \mathrm{m}^{-3}\right)$ is the density and $c_{p}\left(\mathrm{~J} \mathrm{Kg}^{-1} \cdot \mathrm{K}^{-1}\right)$ is the specific heat. One dimension (1D) multilayer model [26] is used for the identification of thermal properties, especially the effective thermal conductivity. As indicated in Figure 7, this 1D model includes the thin film and substrate media connected by a thermal contact resistance at the interface. The working conditions of the multilayer thermal model are well developped in ref [26, 15].

The thermal transducer is a non-transparent medium at $248 \mathrm{~nm}$ (the wavelength of the pump laser) and the IR detector is not collecting the reflected (UV) radiations emitted from the surface of the sample. Thermal losses from the surface are negligible versus the absorbed amount of the laser light [27, 28, 29].

Samples 1 and 2 are tested first in this protocol to check the consistency of the identification model in the case of a semi-infinite or a multilayer sample. In the following studies, the curves of the temporal temperature variation begin at the maximum value of the surface temperature. The surface temperature changes versus time for sc-Si (sample 1) is shown in Figure 8 for a laser fluence of $180 \mathrm{~mJ} \cdot \mathrm{cm}^{-2}$. In this case, the maximum value of temperature after a laser shot is close to $70^{\circ} \mathrm{C}$ and the relaxation one (after a time delay of $400 \mathrm{~ns}$ ) is around an adiabatic temperature of $10^{\circ} \mathrm{C}$. Despite the difficulty of such measurement (highly conductive medium, fast relaxation time and relatively low temperature amplitude) the identification model allows to estimate the thermal conductivity at $135 \pm 20 \mathrm{~W} \cdot \mathrm{m}^{-1} \cdot \mathrm{K}^{-1}$, as indicated (in bold) in Table 2 . Notice that the value of the thermal contact resistance has no physical meaning here because the environment is semiinfinite; it is given here only for the virtually digital performance of the identification model. The values of $\rho \mathrm{c}_{\mathrm{p}}$ correspond to the theoretical data of sc-Si at room temperature.

Shifting for the case of Ti/sc-Si (sample 2), the physical parameters given by the analytical model are very close to those obtained in other studies. The estimated values are $130 \mathrm{~W} \cdot \mathrm{m}^{-1} \cdot \mathrm{K}^{-1}$ for $\kappa_{2}$ and $1.10^{-8} \mathrm{~K} \cdot \mathrm{m}^{2} \cdot \mathrm{W}^{-1}$ for $\mathrm{R}_{\mathrm{th} 2}$. These values are obtained also for a laser fluence of $180 \mathrm{~mJ} \cdot \mathrm{cm}^{-2}$. As expected, the thermal conductivity of sc-Si is not affected by the presence of the Ti upper layer.

A paremetric study is carried on sample 2 in order to evaluate the sensitivity of the model on two thermal parameters $\kappa_{2}$ and $R_{\mathrm{th}}$. This study indicates that changes in $\mathrm{R}_{\mathrm{th}}$ affect strongly the maximum of surface temperature and its relaxation slope (figure 9 a). In this case, it becomes impossible to confuse with the experimental curve for incorrect values. In the case of $\kappa_{2}$, the time relaxation does not change significatively for different values of $\kappa_{2}\left(110-150 \mathrm{~W} \cdot \mathrm{m}^{-1} \cdot \mathrm{K}^{-1}\right)$. On the other side, the maximum of the surface temperature increases to $10^{\circ} \mathrm{C}$ corresponding to a variation of $15 \%$ of the value of $\kappa_{2}$ (figure $9 \mathrm{~b}$ ). These last results evidence that estimation of the substrate themal conductivity $\kappa_{2}$ can be estimated with a precision better than $15 \%$. Notice that this sensitivity analysis is conducted on sample 2, because this is the case of the most difficult to investigate due to the relatively high thermal conductivity of the substrate.

\subsection{Thermal properties determination}

As mentioned before, the macro-Si has an important pore diameter, which makes its surface very rough. The contact between the $\mathrm{W}$ thin film and macro-Si substrate being not perfect, even a low laser fluence may induce the removal of the layer. The mean laser fluence used for thermal characterization is therefore decreased here to $50 \mathrm{~mJ} \cdot \mathrm{cm}^{-2}$. Nevertheless, Figure 10 shows a maximum of the surface temperature close to $140^{\circ} \mathrm{C}$. Combination of bad thermal contact resistance $3.2 .10^{-7} \mathrm{~K} \cdot \mathrm{m}^{2} \cdot \mathrm{W}^{-1}$ and high surface emissivity (close to 0.4 ) leads to this high amplitude. Notice that the calculated value of the macro-Si thermal conductivity is about $73 \mathrm{~W} \cdot \mathrm{m}^{-1} \cdot \mathrm{K}^{-1}$ and it is two times smaller than those of the theoretical value of crystalline silicon $\left(145 \mathrm{~W} \cdot \mathrm{m}^{-1} \cdot \mathrm{K}^{-1}\right)$. The values of the volumetric heat capacity of porous silicon (or any porous media) are calculated taking into account the porosity of the sample by applying a barycenter model according to equation 2 (weighted by porosity):

$$
\left(\rho c_{p}\right)_{\text {eff }}=\left(\rho c_{p}\right)_{S i}(1-\text { porosity })
$$


In Table 2, the experimental results of thermal conductivity and thermal conctact resistance are listed as obtained by the model as well as the values of the volumetric heat capacity introduced in the model. Figure 11 shows the surface temperature changes for Ti/meso-Si (sample 4) for a laser fluence of $150 \mathrm{~mJ} \cdot \mathrm{cm}^{-2}$. The maximum of temperature is about $200^{\circ} \mathrm{C}$ and the temperature relaxation is roughly reached after $1 \mu \mathrm{s}$ duration time. There is no significant differences between the surface temperature of sample 4 before laser interaction and after $1 \mu$ s delay. In opposition to sample 3, this different behavior is explained by a good adhesion of Ti thin film on meso-Si mainly due to the small pores size (see Table 1). As a consequence, the thermal contact resistance is, in this case, comparatively small: $0.1-0.3 \cdot 10^{-8} \mathrm{~K} \cdot \mathrm{m}^{2} \cdot \mathrm{W}^{-1}$. Moreover, this is reinforced by the high emissivity of Ti thin film (0.5). . The theraml conductivity of meso-Si is determined in this work is varying between 50 and $80 \mathrm{~W} \cdot \mathrm{m}^{-1} \cdot \mathrm{K}^{-1}$ depending on the porosity. The porosity is also an important parameter to be taken into account. Thermal conduction of air or any other type of residual gas does not evolve in the same manner depending on the size cavity in where it is confined (micrometer size or smaller). In this case (diameter of pores), there is no general theory that explains thermal changes, and no model can be directly applied in order to determine the thermal conductivity versus pore size.

Since the porosity of meso-Si changes with the thickness, two differents studies are carried on considering the two values of porosity: $15 \%$ and $35 \%$ for a thickness of $10 \mu \mathrm{m}$. Results show that for a porosity of $35 \%$, the value of meso-Si thermal conductivity is about $50 \pm 10 \mathrm{~W} \cdot \mathrm{m}^{-1} \cdot \mathrm{K}^{-1}$ and the value of the thermal contact resistance is $0.3 \cdot 10^{-8} \mathrm{~K} \cdot \mathrm{m}^{2} \cdot \mathrm{W}^{-1}$. In case of $15 \%$ of porosity, as the value of the thermal conductivity increases and becomes $80 \pm 15 \mathrm{~W} \cdot \mathrm{m}^{-1} \cdot \mathrm{K}^{-1}$, and the thermal contact resistance value decreases slightly $\left(0.1 \cdot 10^{-8} \mathrm{~K} \cdot \mathrm{m}^{2} \cdot \mathrm{W}^{-1}\right)$, which is probably due to a better adhesion of the film when the porosity decreases. Wolf et al. have also noticed that the value of thermal conductivity derceases when the porosity of the porous silicon samples elaborated under the same method increases [5]. Figures 12 and 13, show a good agreement between the experimental temporal temperature relaxation versus the analytical model in these two case of porosity.

The value of the thermal conductivity of meso-Si is three times lower than the sc-Si sample having the same dopant type and concentration. Thus, the porosity and the thickness of the etched zone, as well as the morphology and struture of the pores affect significantly the conduction process [6,30]. The value of thermal conductivity determined in this work is in the same order of magnitude of the one detremined by other study using te same elaboration technique and porosity [5].

In the case of macro-Si sample, the detremination of the crystalite size is very difficult, as mentionned before, due the elaboration technique. The alignement and shape of crystallites play an important role in the value of the tehermal conductivity. The thermal conductivity of polycristalline silicon thin films is determined in differents studies and turned out to be about one order of magnitude smaller than the monocrystalline silicon [31, 14] and sometines two order of magnitude [13]. These value cannot be compared to our results du to a big number of different parameters starting with the difference between thin films and substrate, the elaboration technique and also the porosity, size of pores and other morphological without forgetting the purity and grain boundary parameters.

The value of the thermal conductivity of macro-Si is more important $\left(73 \mathrm{~W} \cdot \mathrm{m}^{-1} \cdot \mathrm{K}^{-1}\right)$ of the meso-Si $\left(50 \mathrm{~W} \cdot \mathrm{m}^{-1} \cdot \mathrm{K}^{-1}\right)$ one in case of a same porosity (about $\left.35 \%\right)$. The larger size of pores and their random surface distribution makes the macro-Si slightly more conductive than the meso-Si sample.

\section{Conclusion}

In this work, the thermal conductivity of porous silicon was determined by the pulsed-photothermal method. Two samples with different porosity were studied. The effect of porosity as well as the size of pores on the thermal conductivity is clearly established. In both cases, thermal conductivity of porous samples is found much lower than the sc-Si sample, due to the presence of air acting as insulator in the micro and mesopores. Additionally, it is shown that 1D multilayer thermal model is well adapted to identify the apparent thermal properties, either for continues or porous silicon samples. Estimation of $R_{\text {th }}$ and $\kappa_{2}$ are achieved with variations less than $15 \%$. Future works will concern the effect of porosity on the thermal conductivity with samples having the same pores size, as well as the effect of the presence or not of residual gas. Experiments can be done either under vacuum or by injection of a gas like argon or helium in the hemispherical reactor.

Acknowlegement The "Région CENTRE" is acknowledged for its financial support from the APR Nanotherm. 


\section{Figure captions}

Figure 1: Shematic view of the experimental set-up

Figure 2: Cross section SEM observation of macro-Si (a) prepared by sintering and meso-Si (b) prepared by electrochemical etching

Figure 3: Schematic view of the electrochemical etching process

Figure 4: Cross section SEM observation of Ti/sc-Si (sample 2) deposited by a commercial device PECS (Precision Etching Coating System)

Figure 5: Cross section SEM observation of $\mathrm{W} /$ macro-Si (sample 3) (a) and Ti/meso-Si (sample 4) (b)

Figure $\quad 6$ : IR thermal signal calibration of sample 1 to sample 4

Figure 7: Schematic view of the considered 1D thermal multilayer model

Figure $\quad$ 8:Relaxation of surface temperature of sc-Si (sample 1, circle) and Ti/Sc-Si (sample 2, diamonds) after a laser pulse of $180 \mathrm{~mJ} . \mathrm{cm}^{-2}$ and $150 \mathrm{~mJ} . \mathrm{cm}^{-2}$ respectively, versus model values (continous lines)

Figure $\quad$ 9: Sensibility of the model versus $\mathrm{R}_{\mathrm{th}}(\mathrm{a})$ and $\kappa_{2}(\mathrm{~b})$

Figure 10: Surface temperature changes for W/macro-Si (sample 3) for a laser fluence of $50 \mathrm{~mJ} \cdot \mathrm{cm}^{-2}$

Figure 11: Variation of surface temperature of Ti/meso-Si (sample 4) after a laser pulse, at $150 \mathrm{~mJ}^{\mathrm{m}} \mathrm{cm}^{-2}$

Figure 12: Variation of surface temperature normalized by the equilibrum temperature of $\mathrm{Ti} / \mathrm{meso}-\mathrm{Si}$ (sample 4) with a porosity of $35 \%$ after a laser pulse, at $150 \mathrm{~mJ} . \mathrm{cm}^{-2}$, given by the analytical model (continuous line) confronted to the experimental results (circle)

Figure 13: Variation of surface temperature normalized by the equilibrum temperature of Ti/meso-Si (sample 4) with a porosity of $15 \%$ after a laser pulse, at $150 \mathrm{mJ.cm}^{-2}$, given by the analytical model (continuous line) confronted to the experimental results (circle)

\section{Table captions}

Table 1: Characteristics of samples

Table 2: Thermal conductivity and thermal contact resistance of all samples determined by the identification model (in bold) and the volumetric heat capacity identified by the model and the weighted porosity equation 


\section{List of Figures:}

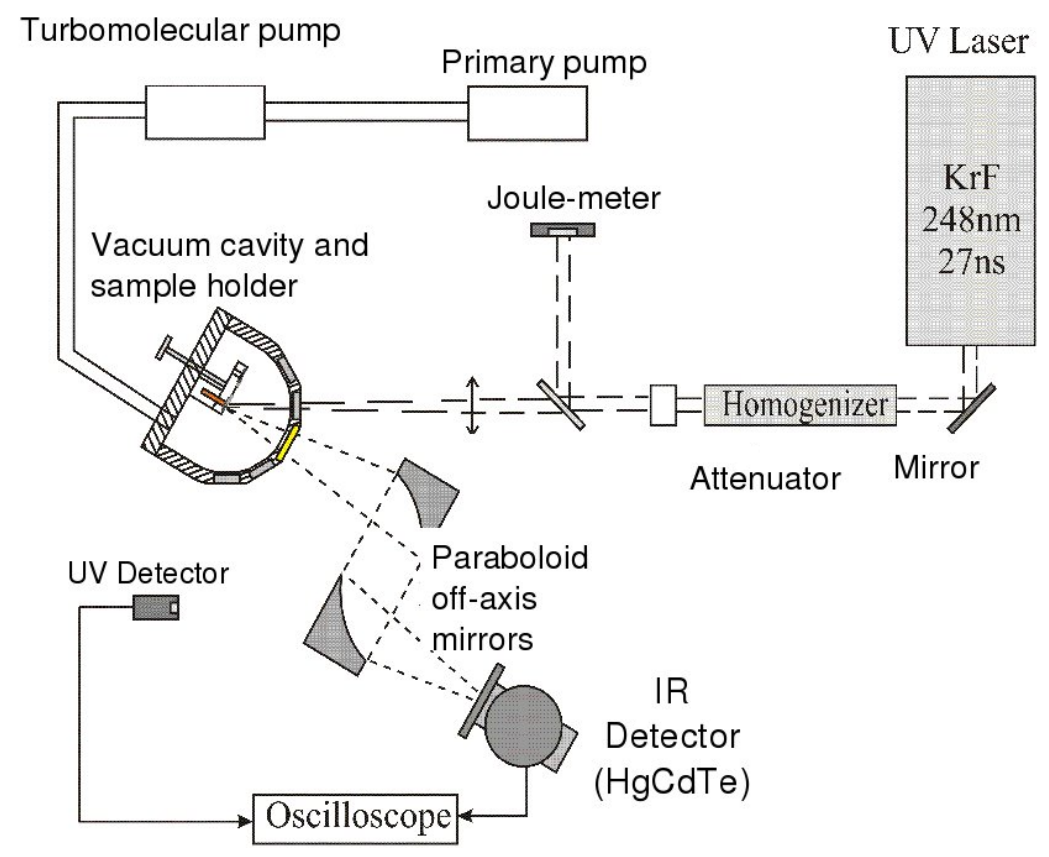

Fig. 1: Shematic view of the experimental set-up

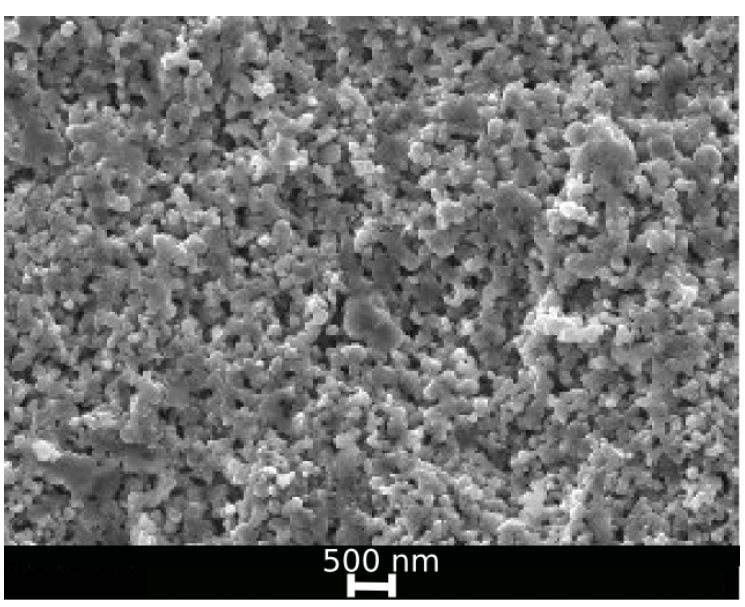

a

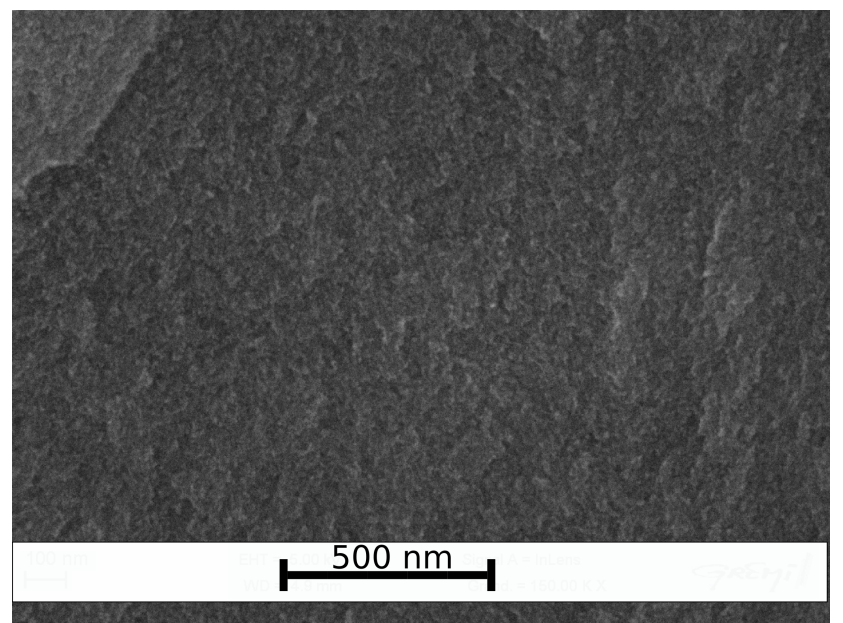

$\mathrm{b}$

Fig. 2: Cross section SEM observation of macro-Si (a) prepared by sintering and meso-Si (b) prepared by electrochemical etching 


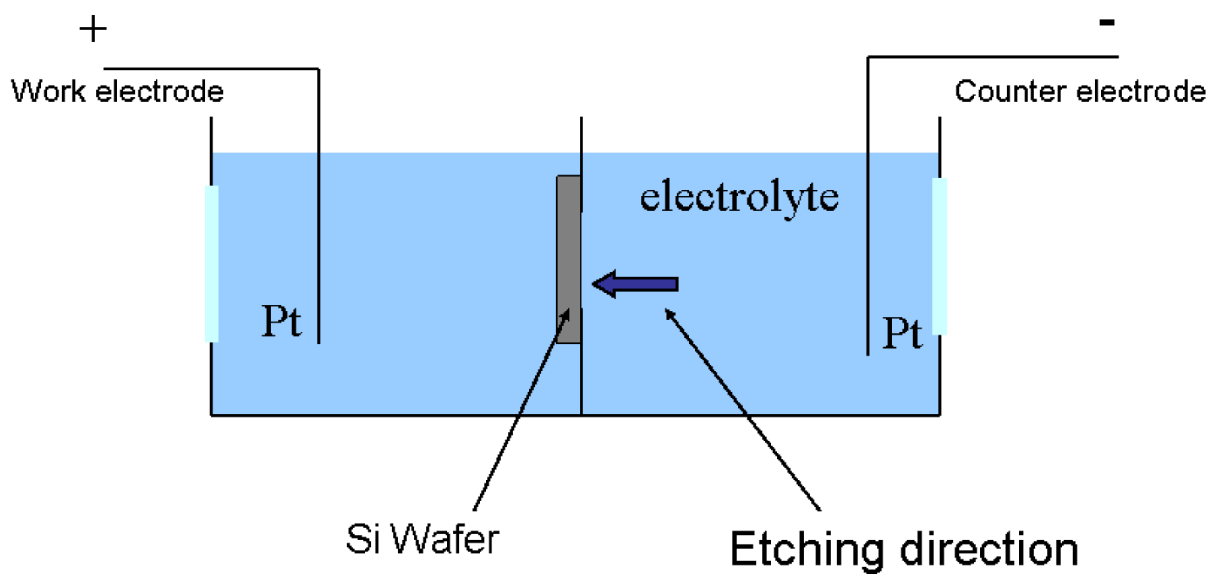

Fig. 3: Schematic view of the electrochemical etching process

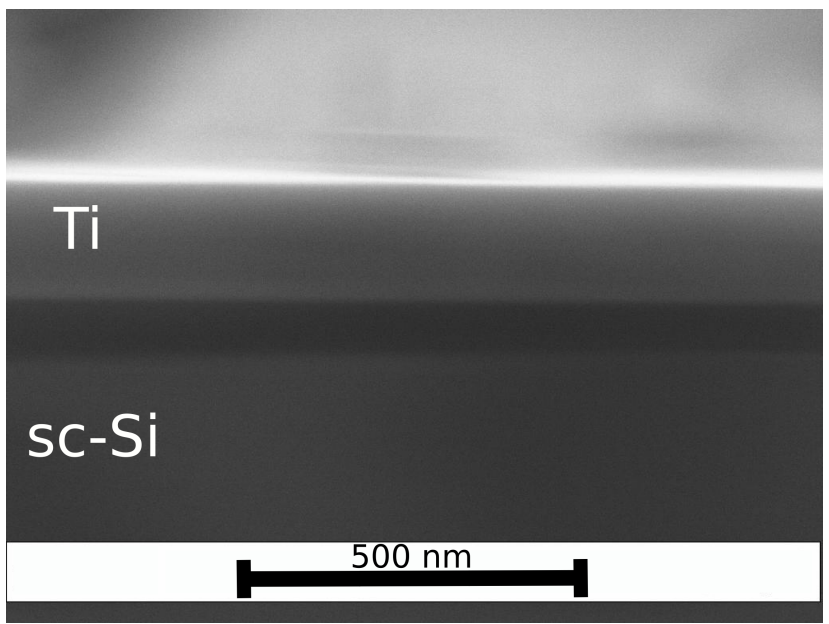

Fig. 4: Cross section SEM observation of Ti/sc-Si (sample 2) deposited by a commercial device PECS (Precision Etching Coating System) 


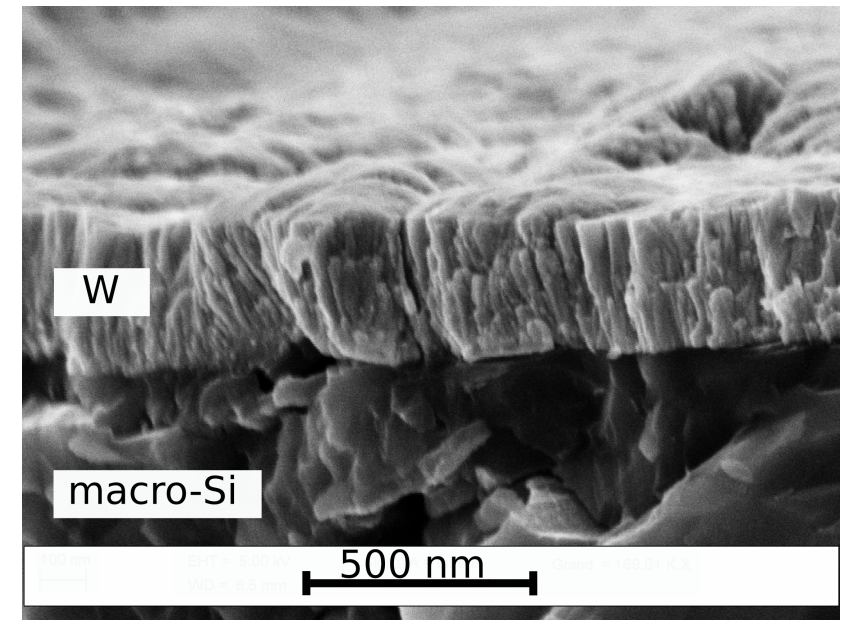

a

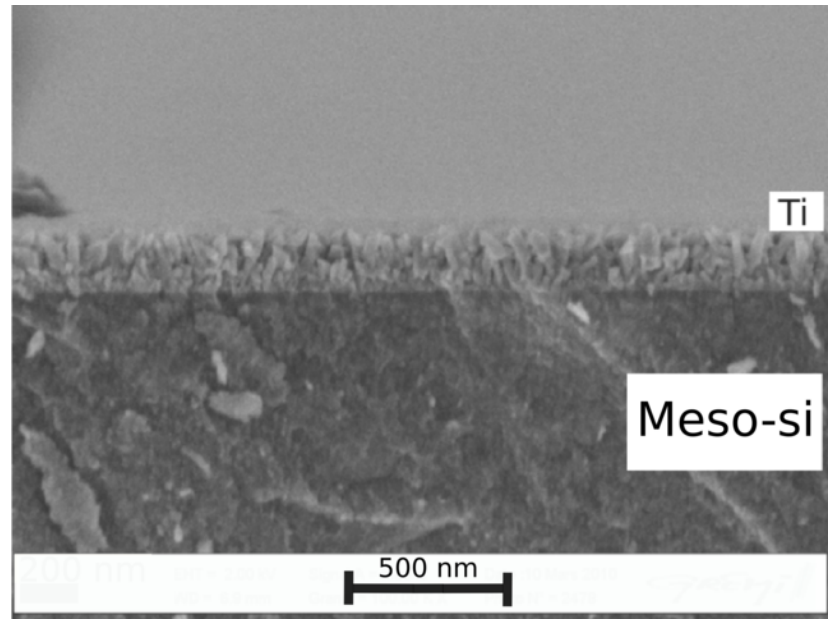

$\mathrm{b}$

Fig. 5: Cross section SEM observation of W/macro-Si (sample 3) (a) and Ti/meso-Si (sample 4) (b)

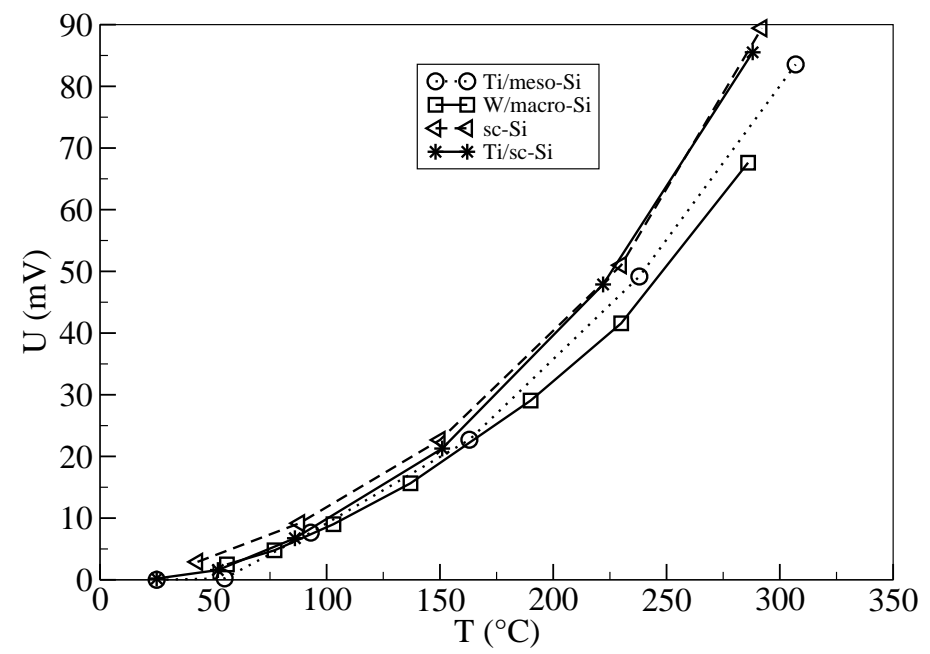

Fig. 6: IR thermal signal calibration of sample 1 to sample 4 


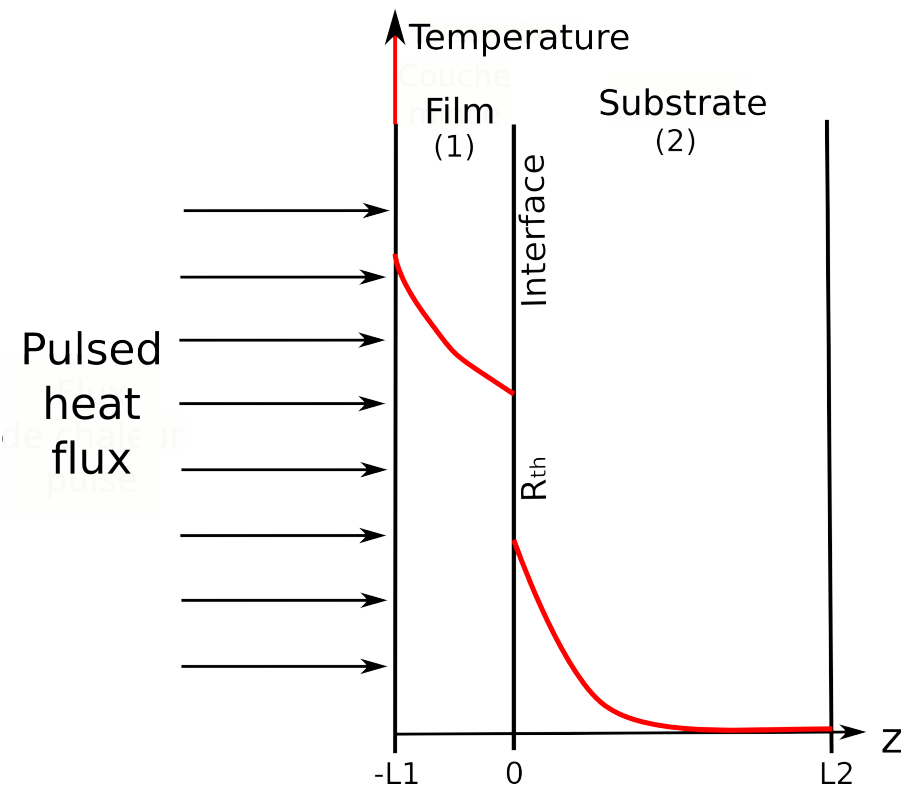

Fig. 7: Schematic view of the considered 1D thermal multilayer model

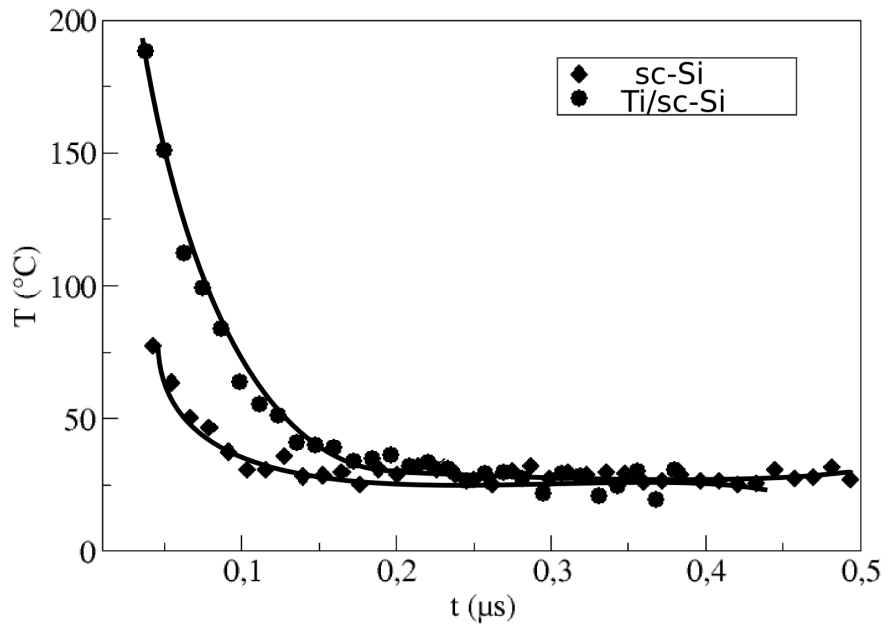

Fig. 8: Relaxation of surface temperature of sc-Si (sample 1, circles) and $\mathrm{Ti} / \mathrm{Sc}-\mathrm{Si}$ (sample 2, diamonds) after a laser pulse of $180 \mathrm{~mJ} . \mathrm{cm}^{-2}$ and $150 \mathrm{~mJ} . \mathrm{cm}^{-2}$ respectively, versus model values (continous lines) 


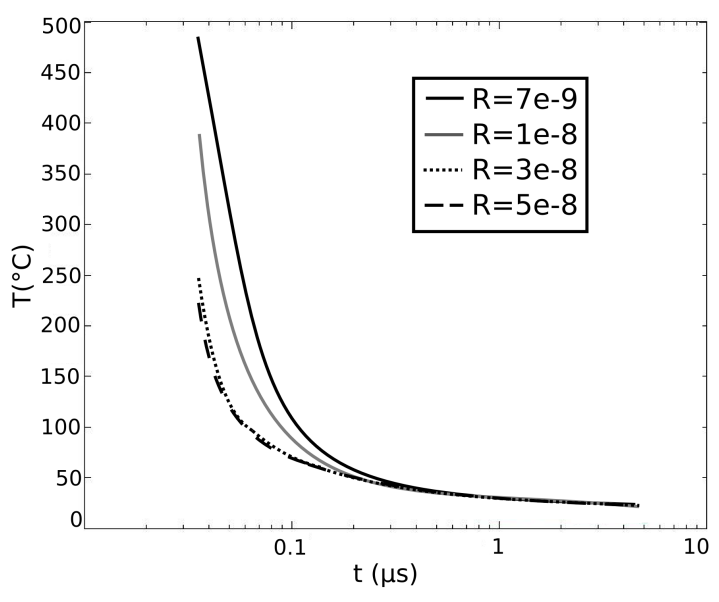

a

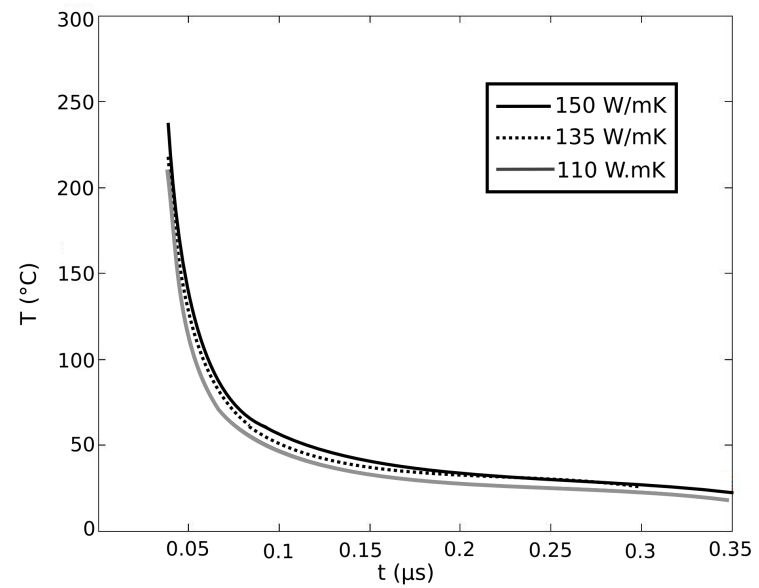

$\mathrm{b}$

Fig. 9: Sensibility of the model versus $\mathrm{R}_{\mathrm{th}}(\mathrm{a})$ and $\kappa_{2}(\mathrm{~b})$

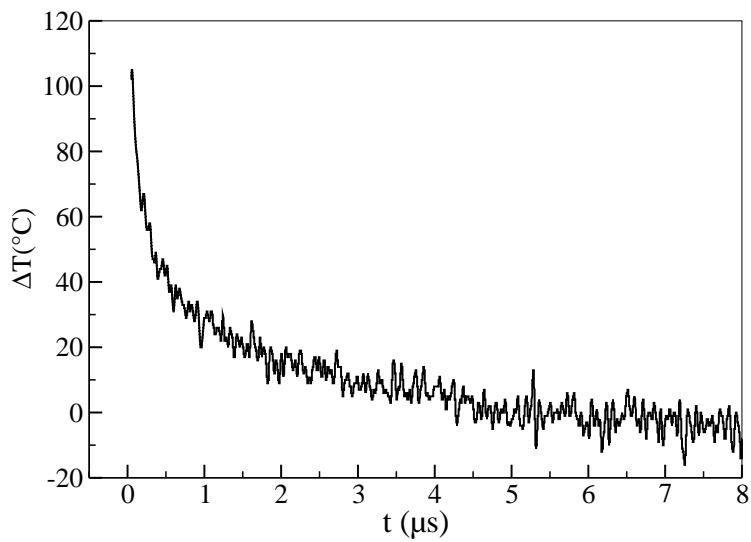

Fig. 10: Surface temperature changes for $\mathrm{W} /$ macro-Si (sample 3) for a laser fluence of $50 \mathrm{~mJ} \cdot \mathrm{cm}^{-2}$ 


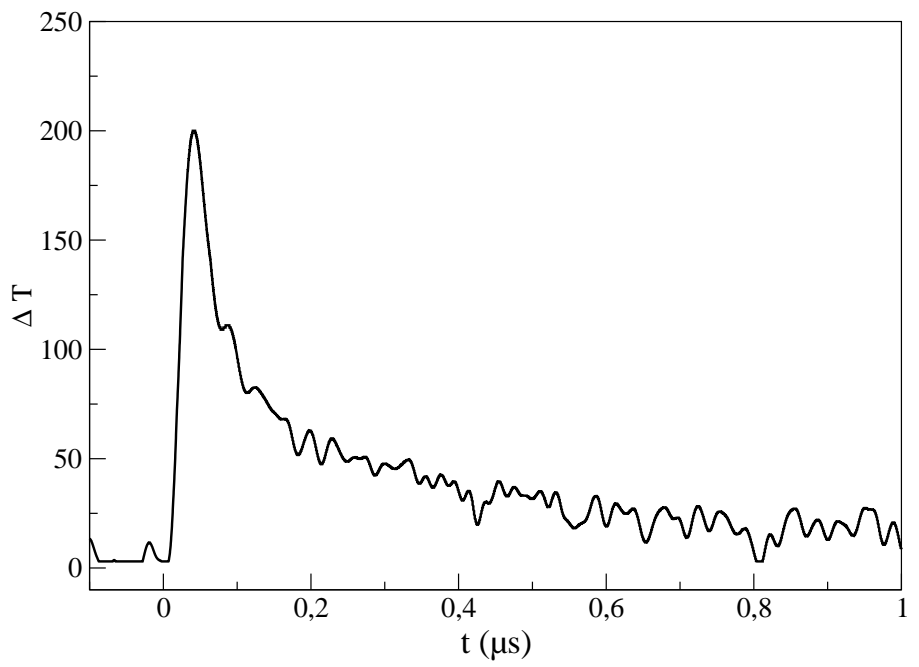

Fig. 11: Variation of surface temperature of $\mathrm{Ti} / \mathrm{meso}-\mathrm{Si}$ (sample 4) after a laser pulse, at $150{\mathrm{~mJ} . \mathrm{cm}^{-2}}^{-2}$

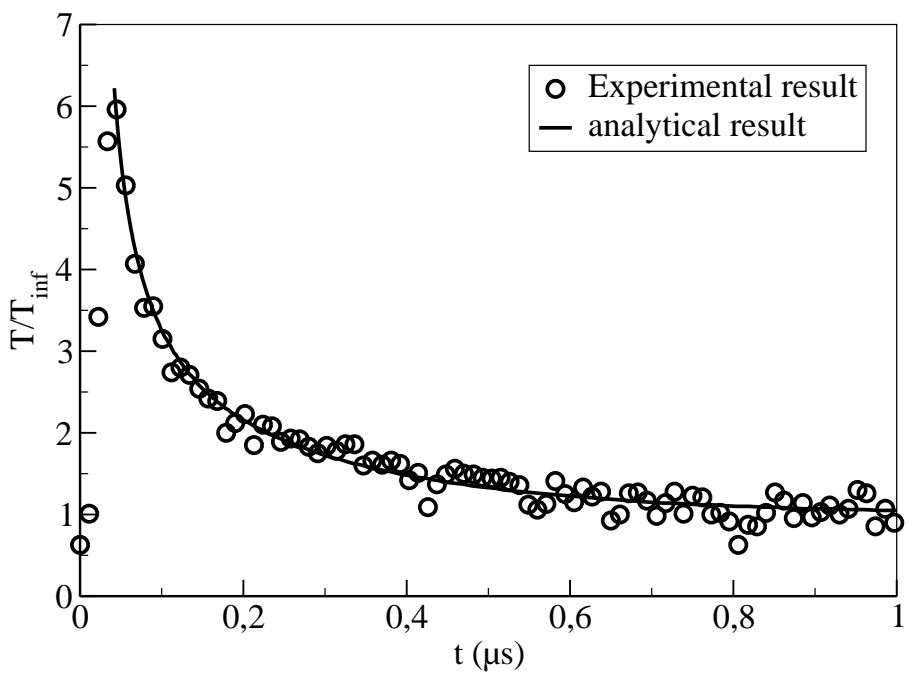

Fig. 12: Variation of surface temperature normalized by the equilibrum temperature of $\mathrm{Ti} / \mathrm{meso}-\mathrm{Si}$ (sample 4 ) with a porosity of $35 \%$ after a laser pulse, at $150 \mathrm{~mJ} . \mathrm{cm}^{-2}$, given by the analytical model (continuous line) confronted to the experimental results (circle) 


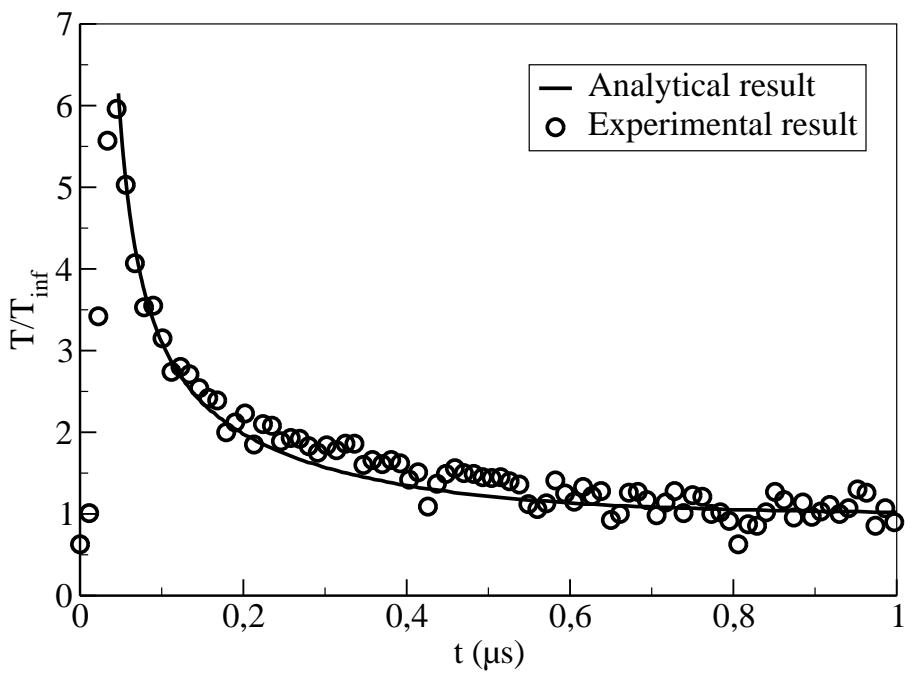

Fig. 13: Variation of surface temperature normalized by the equilibrum temperature of $\mathrm{Ti} / \mathrm{meso}-\mathrm{Si}$ (sample 4 ) with a porosity of $15 \%$ after a laser pulse, at $150 \mathrm{~mJ} . \mathrm{cm}^{-2}$, given by the analytical model (continuous line) confronted to the experimental results (circle) 


\section{List of tables}

\begin{tabular}{|c|c|c|c|c|}
\hline & Sample 1 & Sample 2 & Sample 3 & Sample 4 \\
\hline \hline Type & sc-Si & $\mathrm{Ti} / \mathrm{sc}-\mathrm{Si}$ & $\mathrm{W} / \mathrm{macro}-\mathrm{Si}$ & $\mathrm{Ti} / \mathrm{meso}-\mathrm{Si}$ \\
\hline Thickness & $500 \mu \mathrm{m}$ & $200 \mathrm{~nm} / 500 \mu \mathrm{m}$ & $300 \mathrm{~nm} / \mu \mathrm{m}$ & $600 \mathrm{~nm} / 120 \mu \mathrm{m}$ \\
\hline Substrate pore sizes ()nm & - & - & $100-1000$ & $5-10$ \\
\hline Method of film deposition & - & Ion beam PVD (PECS) & Magnetron sputtering & Magnetron sputtering \\
\hline
\end{tabular}

Tab. 1: Characteristics of samples

\begin{tabular}{|c|c|c|c|c|c|c|c|c|}
\hline & Sample 1 & \multicolumn{2}{|c|}{ Sample 2} & \multicolumn{2}{|c|}{ Sample 3} & \multicolumn{3}{|c|}{ Sample 4} \\
\hline & Sc-Si & $\overline{\mathrm{Ti}}$ & Sc-Si & $\overline{\mathrm{W}}$ & macro-Si & $\overline{\mathrm{Ti}}$ & meso-Si(35\%) & meso-Si(15\%) \\
\hline$\kappa\left(W \cdot m^{-1} \cdot K^{-1}\right)$ & $\mathbf{1 3 5}$ & 30 & 130 & 200 & 73 & 22 & $\mathbf{5 0} \pm \mathbf{1 0}$ & $80 \pm 15$ \\
\hline$\rho c_{p}\left(J \cdot K^{-2} \cdot m^{-3}\right) \cdot 10^{6}$ & 1.5 & 2.23 & 1 & 2.57 & 1.00 & 2.00 & 1.06 & 1.5 \\
\hline$R_{t h}\left(K \cdot m^{2} \cdot W^{-1}\right) \cdot 10^{-8}$ & $10^{-2}$ & \multicolumn{2}{|c|}{1} & \multicolumn{2}{|r|}{32} & \multicolumn{2}{|r|}{0.3} & 0.31 \\
\hline
\end{tabular}

Tab. 2: Thermal conductivity and thermal contact resistance of all samples determined by the identification model (in bold) and the volumetric heat capacity identified by the model and the weighted porosity equation 2 


\section{References}

[1] P. M. Fauchet, "Photoluminescence and electroluminescence from porous silicon," Journal of Luminescence, vol. 70, pp. 294-309, 1996.

[2] H. Hamadeh, M.Naddaf, and A. Jazmati, "Near infrared photoluminescence properties of porous silicon prepared under the influence of light illumination," J. Phys. D: Appl. Phys., vol. 41, p. 245108 (5pp), 2008.

[3] H. Wong, V. Filip, C. Wong, and P. Chung, "Silicon integrated photonics begins to revolutionize," Microelectronics Reability, vol. 47, pp. 1-10, 2007.

[4] E. Guillermain, "Dispositifs nanophotoniques à ondes de surface en silicium poreux : Technologie et application à la bio-détection," Ph.D. dissertation, Ecole doctorale Matériaux De Lyon, 2007.

[5] A. Wolf and R. Brendel, "Thermal conductivity of sintered porous silicon films," Thin Solid Films, vol. 513, pp. 385-390, 2006.

[6] V. Lysenko, V. Gliba, V. Strikha, A. Dittmar, G. Delhomme, P. Roussel, D. Barbier, N. JaffrezicRenault, and C. Martelet, "Nanoscale nature and low thermal conductivity of porous silicon layers," Applied Surface Science 123/124 (1998) 458-461, vol. 123/124, pp. 458-461, 1998.

[7] G. Kaltsas and A. Nassiopoulou, "Novel c-mos compatible monolithic silicon gas flow sensor with porous silicon thermal isolation," Sensors and Actuators, vol. 76, pp. 133-138, 1999.

[8] M. Boutchich, K. Ziouche, P. Godts, and D. Leclercq, "Characterization of phosphorus and boron heavily doped lpcvd polysilicon films in the temperature range 293-373 k," IEE Electronic Device Letter, vol. 23, pp. 139-141, 2002.

[9] J. Randrianalisoa and D. Baillis, "Prédiction de la conductivité thermique des films de silicium poreux," in Thermique des Nanosystèmes et Nanomatériaux ESPCI, 2007.

[10] V. S.-Y. Lin, K. Motesharei, K.-P. S. Dancil, M. J. Sailor, and M. R. Ghadiri, "A porous silicon-based optical interferometric biosensor," Science, vol. 278, pp. 840-843, 1997.

[11] P. E. Raad, P. L. Komarov, and M. G. Burzo, "Thermal characterization of embedded electronic features by an integrated system of ccd thermography and sefl-adaptative numerical modeling," Microelectronics Journal, vol. 39, pp. 1008-1015, 2008.

[12] g. K. Pavel L. Komarov, Nihai G. Burzo and P. E. Raad, "Transient thermo-reflectance measurements of the thermal conductivity and interface resistance of metallized natural and isotopically-pure silicon," Microelectronics Journal, vol. 34, pp. 1115-1118, 2003.

[13] M. L. Seungjae Moon, Mutsuko Hatano and C. P. Grigoropoulos, "Thermal conductivity of amorphous silicon thin films," International Journal of Heating and Mass Transfer, vol. 45, pp. 2439-2447, 2002.

[14] S. Uma, A. McConnell, M. asheghi, K. Kurabayashi, and K. Goodson, "Temperature-dependant thermal conductivity of undoped polycrystalline silicon layers," International Journal of Thermophysics, vol. 22, pp. 605-616, 2001.

[15] J. Martan, N. Semmar, C. Leborgne, E. L. Menn, and J. Mathias, "Thermal properties characterization of conductive thin films and surfaces by pulsed lasers," Applied Surface Science, vol. 247, pp. 57-63, 2005.

[16] J. Martan, N. Semmar, C. Leborgne, P. Plantin, and E. L. Menn, "Thermal characterization of tungsten thin films by pulsed photothermal radiometry," In Nanoscale and Microscale Thermophysical Engineering, vol. 10, pp. 333-344, 2006.

[17] R. Dussart, X. Mellhaoui, T. Tillocher, P. Lefaucheux, M. Boufnichel, and P. Ranson, "The passivation layer formation in the cryo-etching plasma process," Microelectronic Engineering, vol. 84, pp. 1128-1131, 2007. 
[18] G. Gautier, P. Leduc, J. Semai, and L. Ventura, "Thick microporous silicon isolation layers for integrated rf inductors," phys. stat. sol. (c), vol. 5, pp. 3667-3670, 2008.

[19] D. Brumhead, L. Canham, D. Seekings, and P. Tufton, "Gravimetric analysis of pore nucleation and propagation in anodised silicon," Electrochimica Acta, vol. 38, pp. 191-197, 1993.

[20] K. Sing, D. Everett, R. Haul, L. Moscou, R. Pierotti, J. Rouquerol, and T. Siemieniewska, "Reporting physisorption data for gas/solid systems with special reference to the determination of surface area and porosity," Pur Appl. Chem., vol. 57, pp. 603-619, 1985.

[21] V. Lehmann, R. Stengl, and A. Luigart, "On the morphology and the electrochemical formation mechanism of mesoporous silicon," Mat. Sc. Eng. B, vol. 69/70, p. 11, 2000.

[22] X. G. Zhang, Electrochemistry of silicon and its oxide, K. Academi, Ed. Plenum Publishers, 2001.

[23] G.-B. Xu and Q.-A. Huang, "An online test microstructure for thermal conductivity of surface micromachined polysilicon thin films," Sensors Journal, IEEE, vol. 6, pp. 428-433, 2006.

[24] F. X. Alvarez, D. Jou, and A. Sellitto, "Pore-size dependence of the thermal conductivity of porous silicon: A phonon hydrodynamic approach," Applied Physics Letters, vol. 97, pp. 033 103-1-3, 2010.

[25] J. Wang, hong bong jiang, W.-C. Wang, and J. B. Zheng, "Efficient infrared up conversion luminescence in porous silicon: A quantum confinement induced effect," Physical Review Letters, vol. 69, pp. 3252$3255,1992$.

[26] D. Balageas, J. Krapez, and P. Cielo, "Pulsed photothermal modeling of layered materials," J. Appl. Phys., vol. 59, pp. 348-357, 1986.

[27] N. Semmar and C. Boulmer-Leborgne, "Metallic thin films heated by pulsed lasers. numerical simulation of the thermal field and the melting kinetics," J. Phys. IV, vol. 120, pp. 413-420, 2003.

[28] J. Martan, N. Semmar, and C. Boulmer-Leborgne, "Optical system for fast ir radiometry: view factor and normal emissivity investigation," Int. J. Thermophysics, vol. 28, pp. 1342-1352, 2007.

[29] N. Semmar, C. Georges, and C. Boulmer-Leborgne, "Thermal behaviour of electric connector coating irradiated by a laser beam," Microelctronics Journal, vol. 33, pp. 705-710, 2002.

[30] L.-C. Liu and M.-J. Huang, "Thermal conductivity modeling of micro- and nanoporous silicon," International Journal of Thermal Sciences, vol. 49, pp. 1547-1554, 2010.

[31] A. Jain and A. Anil Kumar, "Effect of surface and grain boundary on lattice thermal conductivity of polycrystalline silicon layers," in Internation Symposium of Reaserch Students on Materials Science and Engeneering, 2004. 